\title{
A Wireless Strain Sensor System for Bladder Volume Monitoring
}

\author{
Hung Cao, Shreyas K. Thakar, Timothy Fu*, Manthan Sheth, Matthew L. Oseng, \\ Vaibhav Landge, Young-sik Seo and J.-C. Chiao \\ Department of Electrical Engineering, The University of Texas at Arlington, Arlington, TX, USA \\ * Texas Academy of Mathematics and Science, Denton, and Centennial High School, Frisco, TX, USA
}

\begin{abstract}
A wireless strain sensor system has been designed to monitor the bladder volume in patients suffering from urinary incontinence. An interdigitated capacitive (IDC) strain sensor was micro-machined from a $127-\mu \mathrm{m}$ thick brass shim with a laser system, followed by an encapsulation process to package the sensor in elastic polydimethylsiloxane (PDMS). A proof-ofconcept passive telemetry platform was developed to employ the sensor in vivo and a commercial wireless module was utilized for networking and data recording. The system includes a transducer implant, an external wearable unit and a base station. The implant harvests electromagnetic energy from the external unit, supplies power to operate the sensor and transduces the sensor data back. The wearable unit processes and transfers the signals to the base station connected to a computer which continuously displays and records the strain sensor data on the bladder. The sensor was calibrated and the entire system was tested with a bladder phantom model. Results were promising and demonstrated the feasibility of our passive wireless strain sensing system for urinary incontinence management.
\end{abstract}

Index Terms - Bladder monitoring, interdigitated capacitive strain sensor, urinary incontinence, passive wireless system.

\section{INTRODUCTION}

Urinary incontinence (UI) is the involuntary loss of urine due to bladder control dysfunctions. 25 million adult Americans experience transient or chronic UI and among whom 9-13 million patients have severe symptoms according to the National Association for Continence. Besides the inconvenience and discomfort, urinary incontinence may lead to serious problems such as kidney failure. Dysfunctions in urinary bladder cut off the sensory feedback to the central nervous system making the patient incapable of knowing when to induce urination and void the bladder. In long term, this can cause urine to flow back into the kidneys, resulting in kidney damage and thus the need of hemodialysis for patients [1-2].

Various methods to manage the bladder voiding have been proposed to monitor the bladder volume with sensors or noninvasive measurements [2-3]. Catheterization has been used to sense the bladder volume, but it is highly invasive, inconvenient and may lead to bladder infection. It has been proposed that a pressure sensor mounted between the walls of the bladder to detect the pressure before and after voiding in order to determine the bladder volume. However, it was not reliable due to motion artifacts from patients and in some cases the urinary bladder has an ability to maintain similar pressures before and after voiding [2]. Ultrasound measurement has been widely used to detect the overall bladder volume by the nonlinear wave distortion from water and tissues. However, the bulkiness of measurement instrumentation prevents patients from comfortable, portable and continuous uses. Bladder volume could be measured with bioelectric impedance. Nevertheless, it may irritate bladder muscle and cause unnecessary stimulation to the bladder wall tissues. Electroneurogram (ENG) measurements may be able to detect directly bladder sensory signals but a functional portable device has not yet been developed since it requires complex signal processing and recognition [2].

The bladder walls with mucosa, sub-mucosa, detrusor muscle and serosa layers are elastic and the strain information in the serosa tissue can be correlated to the bladder volume. Resistive-based strain sensors attached to the bladder wall to measure the urine volume have been demonstrated [2-3]. However, they were bulky in size and lacked the integration with a wireless transduction system.

In this work, we developed a sensitive strain sensor integrated with a passive transduction mechanism and a wireless system to monitor in vivo the bladder volume in a patient continuously. The strain sensor is based on variable capacitance employing an interdigitated finger metal structure encapsulated in biocompatible polymers. The entire system consists of three components: an implanted transponder; a wearable unit that transfers electromagnetic energy to the implant, receives the sensor signals from the implant and processes the signals in a microprocessor before sending out wirelessly; and a base station receiving and recording the wireless data. Fig. 1 shows the concept of our bladder volume monitoring system. The implant does not require batteries since it harvests the energy from the wearable unit. The wearable unit has a replaceable battery pack and ports to access the data directly.

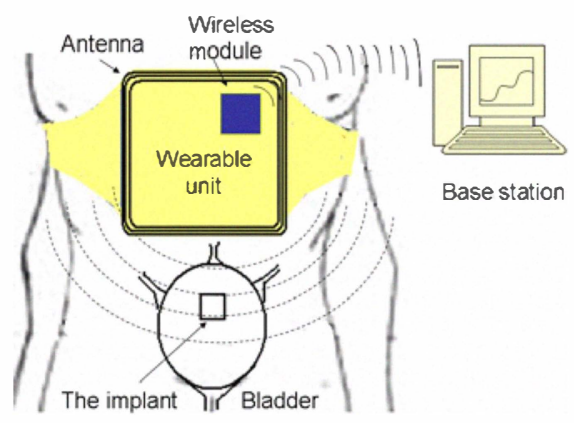

Fig. 1. Wireless system for bladder volume monitoring. The belt is worn at the waist of the patient. In this conceptual drawing, the belt is moved upward in order to show the implant location on the bladder. The sizes of implant and wearable unit are enlarged for illustration purpose. 


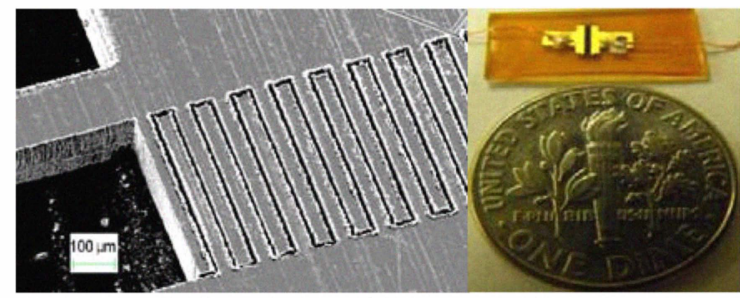

(a)

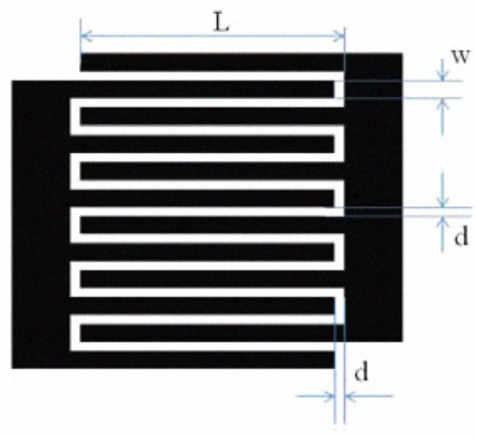

(b)

Fig. 2. (a) SEM photo of the IDC and a photo of the encapsulated sensor. (b) Layout of the IDC.

\section{DESIGN AND FABRICATION}

\section{A. IDC strain sensor}

The IDCs were made with a $127-\mu \mathrm{m}$ thick brass shim by a programmable 355-nm 5-W Oxford laser micro-machining system. The design was sketched by Alphacam software and translated into a $G$-code program. The finger length is $500 \mu \mathrm{m}$ and the separation gaps are $5 \mu \mathrm{m}$. There were total 30-pair fingers in our design. The IDC cutout was attached onto a thin, half-cured PDMS (Sylgard 184) layer which was spun on a 50- $\mu \mathrm{m}$ thick polyimide film. After the PDMS was cured, wire connections were made, followed by casting a top layer of PDMS to encapsulate the entire sensor. An SEM photo of the IDC element and a photo of the strain sensor are shown in Fig. 2 (a). Fig. 2 (b) shows the layout of the IDC.

The capacitance of the IDC can be expressed as

$$
C_{I D C} \propto \frac{\varepsilon L t \alpha \beta N}{d}
$$

where $\varepsilon$ is the effective permittivity of the package including PDMS, polyimide and air; $L$ is the finger length; $t$ is the thickness of the brass shim; $d$ is the separation gap, $N$ is the number of the finger pairs; $\alpha$ and $\beta$ are the terms related to the thickness of polymers and fringe effects of electric fields. As the polymer is stretched, the gaps between the fingers $(d)$ increase inducing the capacitance decrease. The IDC capacitors in our design at the zero-strain state have values in the range of $12-15 \mathrm{pF}$ measured by an LCR meter at a bias voltage of $1 \mathrm{~V}$ and a frequency of $100 \mathrm{kHz}$.

\section{B. System design}

The system blocks are illustrated in Fig. 3. The RF powering from the wearable unit to the implant utilized an operating frequency of $1.3 \mathrm{MHz}$ with a duty cycle of $30 \%$. The sensor signal transduction was conducted with loadmodulating at the same operating frequency.

The communication between the wearable unit and the base station was based on two eZ430RF2500 (Texas Instrument) modules. This module has an embedded MSP430 microcontroller, a 10/12 bit ADC, a CC2500 transceiver chip and a small on-chip antenna. The programmable CC2500 transceiver which has the working frequency range of 2400-2483.5 MHz supports a sleep mode (400 nA current consumption) with fast start-up time ( $240 \mu$ s from sleep to transmit or receive modes). The wireless communication utilizes SimpliciTI which is a standard RF network protocol. This simple and low-power protocol is capable for both star and peer-to-peer networks. The wireless communication range is up to $35 \mathrm{~m}$ in normal situations.

\section{(1) The implant}

The passive implant consists of a coil antenna $\left(\mathrm{L}_{2}\right)$, a 1-nF capacitor $\left(\mathrm{C}_{2}\right)$, a switch, an energy harvesting circuit, a relaxation oscillator and the IDC sensor. A 24-turn coil antenna made of 30AWG magnet wires has a size of $1 \times 2 \mathrm{~cm}^{2}$ resulting in a value of $15 \mu \mathrm{H}$, forming a resonance with $\mathrm{C}_{2}$. The resonance frequency is also at $1.3 \mathrm{MHz}$ so the power received in $\mathrm{L}_{2}$ is maximized.

A voltage multiplier was utilized with a series of diodes and capacitors to increase the transduction distance [5]. The output of the 4-stage voltage multiplier was connected to a $2.5-\mathrm{V}$
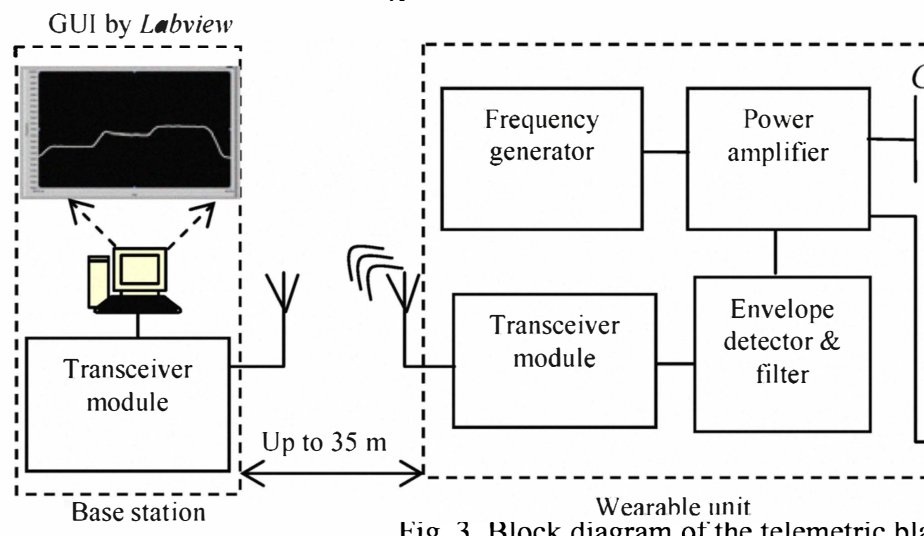

Fig. 3. Block diagram of the te

RF powering

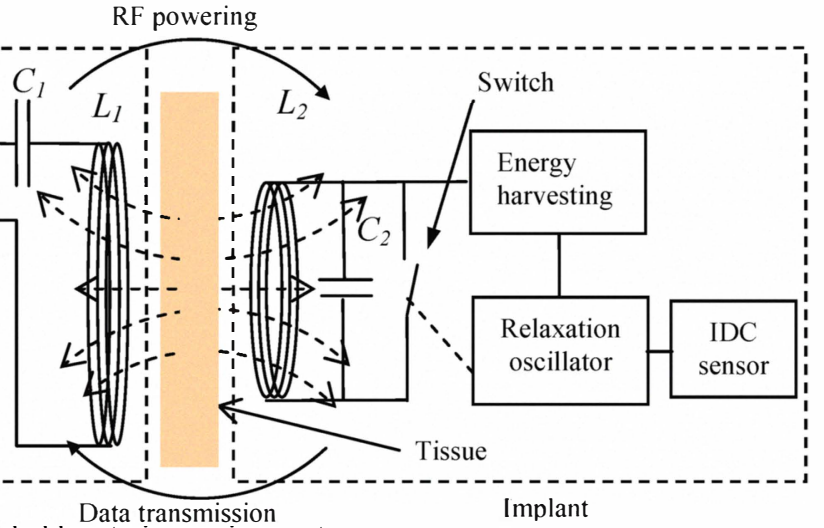

er strain sensing system. 


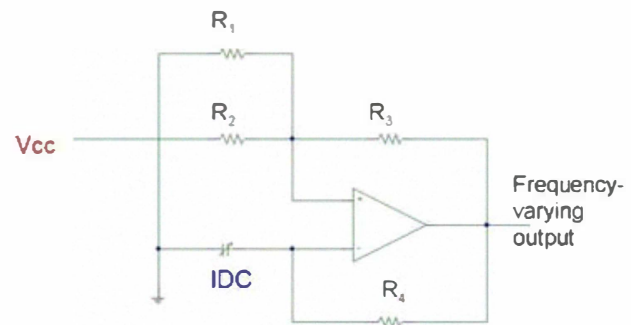

Fig. 4. Relaxation oscillator circuit in the implant.

CMOS regulator which consumed current less than $2 \mu \mathrm{A}$. A $12-\mathrm{V}$ Zener diode was added to protect the regulator from high voltages in case the transponder is very close to the reader. A $1-\mu \mathrm{F}$ storage capacitor was placed at the regulator output to keep the voltage stable during modulation.

A relaxation oscillator circuitry was used to convert the changes in capacitance of the IDC sensor to frequency variations. A low-power op-amp LPV321 (Texas Instrument) was used as a comparator. The resistors $R_{1}, R_{2}, R_{3}$, and $R_{4}$ were $1 \mathrm{M} \Omega, 1 \mathrm{M} \Omega, 1 \mathrm{M} \Omega$ and $2.5 \mathrm{M} \Omega$, respectively (Fig. 4). The frequency generated can be calculated as

$$
f=\frac{1}{2 \ln (3) R_{4} C_{I D C}}
$$

and with the designed $C_{I D C}$ values, the modulated frequency is in the range of $12-15 \mathrm{kHz}$.

\section{(2) The wearable unit}

The 7-turn coil antenna $\left(\mathrm{L}_{1}\right)$ was made from magnet wires wound around a foam board. It has a dimension of $12 \times 15 \mathrm{~cm}^{2}$ giving an inductance of $17 \mu \mathrm{H}$. The capacitor $\mathrm{C}_{1}$ was chosen to be $900 \mathrm{pF}$ for resonance at $1.3 \mathrm{MHz}$. The wearable unit includes a class-E power amplifier which generates high voltages across the coil antenna for electromagnetic fields coupling into the implant; an envelope detector to read the load-modulation signals; and a wireless transceiver to send the sensor data to the base station [6].

The frequency generator provides the operating frequency signals feeding to the amplifier. The source is adjusted to 1.3 $\mathrm{MHz}$ matching the resonant frequency. At the implant, loadmodulation by the relaxation oscillator, in which the frequency was controlled by the IDC capacitance, varies the voltage levels at the reader coil antenna. The modulated signals are extracted by an envelope detector and fed through a band-pass filter to suppress the high frequency carrier data. The sensor signals are then amplified and fed to the input port of the microcontroller in the wireless module.

The MSP430 in the wireless module was programmed to receive the modulated-frequency signals by counting the number of pulses in a duration of $0.25 \mathrm{~s}$. The modulatedfrequency is loaded into the data packet and transmitted wirelessly to the base station.

\section{(3) Base station}

The base station consists of an eZ430RF2500 module and interfaces. The received data was sent to a computer by the MSP430 microcontroller through a serial port and displayed in real time with a graphical user interface (GUI) programmed in Labview. The data is also logged into text files for off-line diagnosis.

\section{EXPERIMENTS AND RESULTS}

Experiments were conducted at a distance of $10 \mathrm{~cm}$ in air between the coil antennas of the wearable unit and implant. For the carrier signal of a square waveform with an amplitude of $4.1 \mathrm{Vpp}$ at $1.3 \mathrm{MHz}$, the signal to noise ratio (SNR) of the transduced signals from the implant remained to be better than $30 \mathrm{~dB}$. With a bag filled with saline solution mimicking thick tissues between the coils, communication distance would be reduced to $5 \mathrm{~cm}$ given the same SNR. By increasing the carrier signal amplitudes to $5 \mathrm{Vpp}$, the transduction distance in air could reach up to $20 \mathrm{~cm}$.

\section{A. Sensor calibration}

The IDC strain sensor was calibrated in a cantilever beam setup (Fig. 5). The sensor was glued with epoxy on top of the cantilever with a distance of $1.76 \mathrm{~cm}$ from the fixed end. The strain at the sensor location can be calculated as [4]

$$
\varepsilon_{x}=\frac{6 P(L-x)}{E b t^{3}}
$$

where $P$ is the load applied at the free end, $L$ is the length of the cantilever, $E$ is the Young's modulus of the cantilever material, $x$ is the distance to the fix end, $b$ is the width and $t$ is the thickness of the cantilever. In the free end of an aluminum cantilever beam with a Young's modulus of $E=69 \times 10^{9} \mathrm{~N} / \mathrm{m}^{2}$ and dimensions of $L=50 \mathrm{~cm}, t=0.63 \mathrm{~cm}$ and $b=1.26 \mathrm{~cm}$, loads were applied in 5 discrete steps with $2.25 \mathrm{~kg}$ each up to 11.25 $\mathrm{kg}$. The corresponding calculated strains were $0.74,1.48$, $2.22,2.96$ and $3.7 \%$. The measured frequency as a function of strain is shown in Fig. 6. The sensitivity was $218.83 \mathrm{~Hz}$ per percentage of strain. The experiment was repeated four times and the results were repeatable and reversible indicating the IDC sensor has not reached the elastic limit.

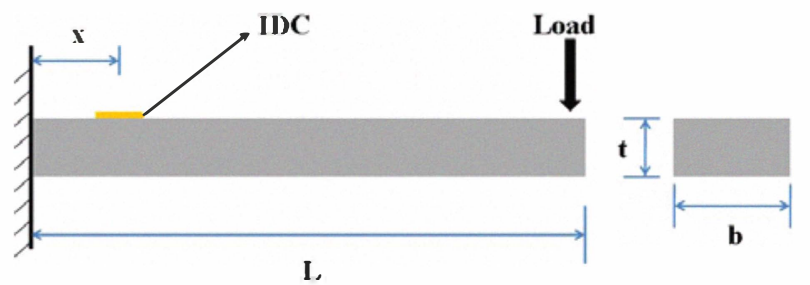

Fig. 5. Cross sectional view of the cantilever beam.

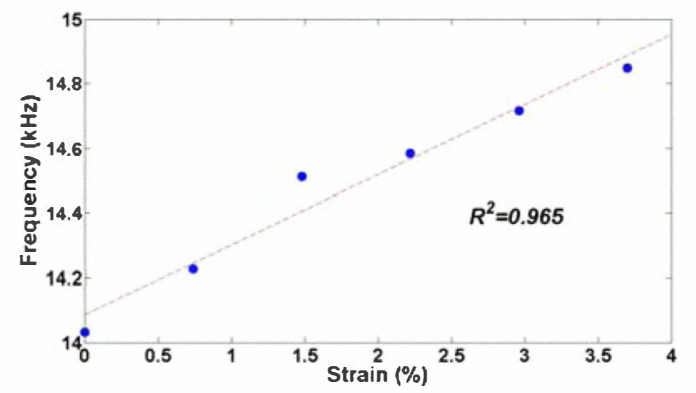

Fig. 6. Sensor calibration results in the cantilever experiment. 


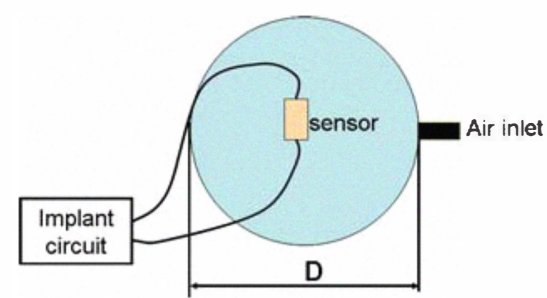

Fig. 7. Experimental setup with the balloon model.

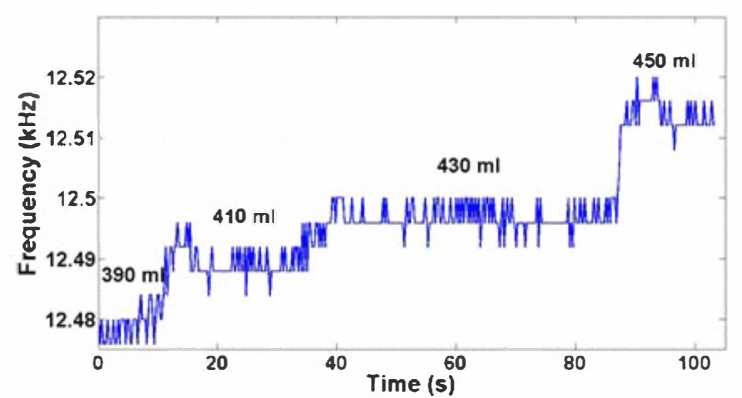

Fig. 8. Real-time measured modulated frequencies for various volumes in the balloon model experiment.

\section{B. Bladder model experiment}

Typically, the maximum volume of a human bladder is 550 $\mathrm{ml}$ and the volume at which patients should trigger voiding is $400 \mathrm{ml}$. Assuming that the bladder is a perfect sphere, its volume can be calculated from the diameter. The IDC strain sensor is designed to be attached on the serosal wall of the bladder. Measurement starts when its volume reaches beyond $390 \mathrm{ml}$. An alert message will be sent out at the $390-\mathrm{ml}$ volume and the modulated frequency output indicates the strain/volume information. As a proof of concept, one sensor was glued on a latex air balloon with medical epoxy. The balloon had an initial diameter $(D)$ of $9.06 \mathrm{~cm}$ which corresponds to a volume of $390 \mathrm{ml}$. Air was pumped in 3 steps with the corresponding volumes of 410,430 and $450 \mathrm{ml}$ while the base station continuously displayed and recorded the modulated frequency. The volume changes corresponded to strains of $1.68,3.31$ and $4.89 \%$, and obtained modulatedfrequencies of $12.487,12.498$ and $12.512 \mathrm{kHz}$, respectively. Fig. 7 shows the experimental setup on the latex balloon, and the real-time data obtained in the base station is shown in Fig. 8. The sensitivity was $8 \mathrm{~Hz}$ per percentage of strain.

\section{DISCUSSIONS AND CONCLUSION}

An IDC strain sensor integrated with a wireless system to monitor bladder volume has been demonstrated. The proof-ofconcept device has shown the capability to measure the volume changes in a bladder model in vivo utilizing inductive coupling and load-modulation signal transduction between the implant and an external wearable unit. Commercial transceivers were implemented in the system to process, relay wirelessly and record the strain data into a computer. The wireless system can be used with multiple sensors to continuously monitor several physiological signals in a patient or several patients in a group wirelessly.

The initial capacitance was reduced when the sensor was attached on the balloon. This was due to the bending of the interdigitated fingers on the curvature of the balloon surface. When the balloon expanded, the capacitance reduced due to strain, but on the other hand the curvature of bending was reduced which increased the capacitance. The opposite effects may not be a practical issue since the sensor will be calibrated after it is implanted to suit individual patient's need.

The real-time fluctuations in frequency outputs were due to the motion artifacts when the air was pumped into the balloon and the resolution limit in the strain detection. By increasing the period to count pulse numbers in MSP430, a more stable output could be achieved. However, it will reduce the response time. It may not be a serious issue considering that it takes minutes for urine production before it enters the bladder. To reduce the fluctuations, the resolution (frequency shift per strain change) could be increased by increasing the frequency range of the relaxation oscillator in the implant.

Our wireless system architecture is capable of monitoring multiple patients and/or multiple sensors simultaneously. In the latter case, the identification information could be embedded in the modulated frequency range for each sensor. The microcontroller could be programmed to multiplex the sensor signals. The transceivers with module IDs in wearable wireless units could represent individual patients.

\section{ACKNOWLEDGEMENT}

The authors would like to thank Intel Corp. for financial support. Timothy Fu was supported by the summer student research experience program in the Texas Academy of Mathematics and Science. Matthew L. Oseng was supported by UT-Arlington I Engage Mentoring program.

\section{REFERENCES}

[1] D. A. Smith, "Urinary Incontinence: Epidemiology, Demographics, and Costs," Incontinence, Vol. 11, 2003.

[2] S. Rajagopalan, M. Sawan, E. Ghafar-Zadeh, O. Savadogo, and V. P. Chodavarapu, "A Polypyrrole-based Strain Sensor Dedicated to Measure Bladder Volume in Patients with Urinary Dysfunction", Sensors, Vol. 8, pp. 5081-5095, 2008.

[3] C. A. Gutierrez and E. Meng, "Low-cost Carbon Thick-film Strain Sensors for Implantable Applications", J. Micromech. Microeng., Vol. 20, 2010.

[4] F. P. Beer and E. R. Johnston, Jr., Mechanics of Materials, McGraw Hill, Third Edition, 2002.

[5] G. Vita and G. Iannaccone, "Design criteria for the RF section of UHF and microwave passive RFID transponders," IEEE $T$. Microw. Theory, Vol. 53, pp. 2978-2990, 2005.

[6] K. Wise, D. Anderson, J. Hetke, D. Kipke, K. Najafi, "Wireless implantable microsystems: high-density electronic interfaces to the nervous system," Proc. IEEE, Vol. 92, pp.76-97, 2004. 